\title{
Ways of probing situated concepts
}

\author{
Ana Sofia Morais, Henrik Olsson, And Lael J. Schooler \\ Max Planck Institute for Human Development, Berlin, Germany
}

\begin{abstract}
Two ways of eliciting conceptual content have been to instruct participants to list the intrinsic properties that concept exemplars possess or to report any thoughts that come to mind about the concept. It has been argued that the open, unconstrained probe is better able to elicit the situational information that concepts contain. We evaluated this proposal in two experiments comparing the two probes with regard to the content that they yield for object concepts at the superordinate and basic levels. The results showed that the open probe was better able to elicit situated conceptual knowledge and point out differences in the representations of superordinate and basic concepts.
\end{abstract}

A concept may be defined as the "accumulated information in memory abstracted for a category, where a category is a set of things in the world perceived as the same type of thing" (Yeh \& Barsalou, 2006, p. 352). Mainstream theories of knowledge incorporate the assumption that concepts are made up of smaller components of meaning representing the properties, or intrinsic characteristics, category exemplars possess. One way of collecting empirical representations with which to test these theories has been to ask people to retrieve properties of concepts from memory by completing sentence stems, such as "a dog is _, has _, can _ " (Garrard, Lambon Ralph, Hodges, \& Patterson, 2001). Properties consist of single words or short phrases that can be connected to the target concept by the word adjoining each blank space. Barsalou and Wiemer-Hastings (2005) raised the concern that this task instruction may not provide an accurate account of human conceptual knowledge. Rather than being context independent, their argument goes, concepts contain extensive information about situations. As an alternative, the authors proposed an open-ended probe that instructed participants to report their thoughts about each concept as they came to mind and confirmed the probe's ability to elicit situational content. Yet it remains to be empirically investigated whether the two retrieval probes-referred to as the property probe and the open probe, respectively - differ in their ability to elicit situational knowledge. The work reported here compared the two task instructions with regard to the content that they elicit for object concepts at the basic (e.g., dog) and superordinate (e.g., mammal) levels.

\section{Is the Open Probe Able to Elicit Situational}

\section{Knowledge Better Than the Property Probe?}

The situated approach to concepts assumes that when the properties of an instance are abstracted, information about the situation is stored as well (Yeh \& Barsa- lou, 2006). It follows that a concept (e.g., car) should contain not only the properties that exemplars possess $(<$ engine $>)$, but also features of situations, such as settings $(<$ jammed highway $>)$, agents $(<$ police officer $>)$, objects $(<$ ambulance $>)$, events $(<$ car accident $>)$, and introspections ( $<$ wish I had a flying car $>) .{ }^{1}$ When asked to retrieve the properties of a concept, "people may scan across many situations, and try to find common properties that fit in the short blanks" (Barsalou \& Wiemer-Hastings, 2005 , p. 154). It follows that the property probe may not allow participants to describe the collection of situations that they may retrieve.

With this concern in mind, Barsalou and WiemerHastings (2005) explored whether an open-ended task instruction, the open probe, would be able to elicit situated conceptual knowledge. In their experiment, participants were not asked to restrict their responses to the properties that concept exemplars possess. Rather, they were told that there were no correct responses to produce and that they should report their thoughts about each concept as they came to mind. Barsalou and Wiemer-Hastings showed that when participants are probed for retrieval in such an unconstrained manner, they retrieve more setting featuresdescribing surrounding spaces, including agents, objects, and events - than properties of the target concepts, with introspective features being the third most frequent. Situated knowledge (aggregating across setting and introspective features) occurred at a combined proportion of .67 , whereas the proportion of properties produced was only .26. This tendency was observed not only for object concepts such as insect, the kind of concepts this article is concerned with, but also for abstract concepts such as truth. Barsalou and Wiemer-Hastings therefore suggested that "open-ended response collection enables more direct measurement of the underlying information stored for a concept" (p. 154). Nevertheless, it may be premature to conclude that one probe enables a more direct measure-

A.S. Morais, morais@mpib-berlin.mpg.de 
ment of concept-related information than does the other without comparing them systematically. Furthermore, the knowledge people retrieve is assumed not to be a verbatim readout of a static definition stored in memory but, rather, "one of many possible temporary abstractions that can be constructed on line" (Barsalou, 2003, p. 1184) in response to a probe. It follows that the knowledge retrieved should be shaped by the particular retrieval probe used. If Barsalou and Wiemer-Hastings's reasoning is correct, the open probe should yield a larger proportion of setting features, as compared with properties, whereas the property probe should yield the reverse pattern. The primary aim of the present work was to evaluate this proposal.

\section{How Do Superordinate and Basic Concepts Differ in Content?}

How does the content of superordinate concepts such as vehicle or reptile differ from that of taxonomically less general, basic concepts such as car or snake $?^{2}$ Superordinate names are most likely to be used as plurals to refer to groups of objects rather than to individual objects (e.g., Callanan, 1985; Wisniewski \& Murphy, 1989). Seldom does one use vehicle to refer to a single bicycle. On the basis of this observation and complementary evidence from the child development literature, Murphy and Wisniewski (1989) suggested a hypothesis about how adult superordinate and basic concepts differ:

Superordinate concepts may contain more relational information: information about the relative locations of exemplars, information about the functional relations between exemplars, and information about how concept exemplars interact with non exemplars. ... Basic concepts, on the other hand, probably emphasize information about the structures and perceptual characteristics of individual objects (p. 573).

That is, whereas superordinates should include more information about multiple objects and their relations in typical situations, basic concepts should include more properties of single objects.

A secondary aim of the present work was to examine potential differences in the knowledge underlying superordinate and basic concepts, using the feature retrieval task. In line with Murphy and Wisniewski's (1989) logic, we expected the proportion of setting features retrieved for superordinates to be larger than the proportion of properties, with the opposite pattern being observed for basic concepts. Yet it may well be that the open and property probes influence the knowledge retrieved for the two concept levels. Thus, an open question arose as to whether an interaction between retrieval probe and concept level would be observed in the experiments described below.

\section{EXPERIMENT 1}

Experiment 1 compared the open and property probes with respect to their ability to elicit the situational content of object concepts. Since the type of probe was manipulated as a between-subjects variable, the open probe con- dition constituted a replication of Barsalou and WiemerHastings (2005) for the particular case of object concepts. The experiment further assessed the hypothesized differences in the knowledge underlying superordinate and basic object concepts.

\section{Method}

Participants and Design. Sixteen participants, with an average age of 24 years, were assigned to one of four between-subjects conditions varying the retrieval probe (open vs. property) and the taxonomic level of the concept (superordinate vs. basic). All the participants were native German speakers and were paid $€ 5$ for taking part in the study. Due to a recording problem, the data from 1 participant were lost.

Materials. Two sets of 11 concrete nouns were used. Each set was composed of 10 nouns sampled from Ruts et al.'s (2004) category and exemplar norms. One set, the superordinate set, included 10 nouns corresponding to superordinate categories in the world (reptile, amphibian, mammal, fish, insect, tool, vegetable, fruit, bird, and vehicle). Each noun in the second set, the basic set, corresponded to the most frequently generated exemplar in each superordinate category, according to Ruts and colleagues' norms (snake, frog, dog, salmon, fly, hammer, lettuce, apple, sparrow, and car, respectively). One additional item was added to each set ( furniture to the superordinate set and sofa to the basic set). Moreover, two items were used for training in each condition: musical instrument and flower in the superordinate condition and guitar and tulip in the basic condition. To control for order effects, two random list orders were established for each set.

Procedure. Half of the participants received the open probe, which instructed them to respond out loud with the thoughts that came to mind about each concept. The other half of the participants received the property probe, which instructed them to list the properties of each concept. Descriptions of the open and property probes are provided in the Appendix. In line with previous studies in which one probe or the other was used, the open probe instruction mentioned that there were no correct features to produce, whereas the property probe instruction omitted whether that was the case or not. In most property norming studies, participants are asked to write down lists of properties (e.g., Garrard et al., 2001), but, in order to equate the two probe conditions with respect to the response format, we asked our participants in the property probe condition to report their knowledge out loud.

Once the instructions had been given, half of the participants retrieved knowledge for superordinate concepts, and the other half for basic concepts. In both probe conditions, the participants first practiced retrieving knowledge for two concepts (musical instrument and flower for the participants in the superordinate condition and guitar and tulip for the participants in the basic condition). After practice, the participants retrieved knowledge for the 11 test items. Following previous studies (e.g., McRae, Cree, Seidenberg, \& McNorgan, 2005), the participants were given as much time as needed, with each concept being presented only when the participants had finished retrieving knowledge for the previous one.

On every test trial, depending on the condition, the participants were asked to list the properties of the target concept or to report their thoughts about it. The participants in the property probe condition were given the following instruction on every trial: "As soon as I say the concept name, please start listing 'is,' 'has,' and 'can' properties of the concept aloud: [concept name]." The participants in the open probe condition, on the other hand, received an instruction adapted from that used by Barsalou and Wiemer-Hastings (2005). In their experiment, participants were given the following probe on every trial: "Please report your thoughts as they come to mind. What characteristics are typically true of the following concept: [concept name]?" One may argue that asking participants to produce characteristics that are typically true of the target concept contradicts the idea, conveyed earlier in the instructions, that there are no correct re- 
sponses to the task. For example, imagine that a participant retrieved the feature $<$ may be taken outside on a leash $>$ when thinking of the concept cat. Under Barsalou and Wiemer-Hastings's instructions, it would be unclear whether the participant should regard such a feature as a valid response or not. On the one hand, since cats are not typically taken outside on a leash by their owners, the participant should consider the feature to be false of the target concept and, therefore, should suppress it. On the other hand, if there are no correct features to produce, any feature one retrieves for a concept constitutes a valid response. To bypass this ambiguity while preserving the open-ended nature of their probe, we asked our participants only to report their thoughts as they came to mind on each trial: "As soon as I say the concept name, please start reporting your thoughts about the concept aloud as they come to mind: [concept name]." When ready, the participants began reporting their thoughts or listing properties, for as long as they could. The participants varied substantially in the time they took to perform the task, ranging from $12 \mathrm{~min}$ to $1 \mathrm{~h}$. Each protocol was digitally recorded.

\section{Results and Discussion}

Each of the 11 audio protocols for a given participant was transcribed into a text file and translated from German into English by a bilingual translator. Because our goal was to compare the content elicited by the property and open probes, and not to construct empirically derived conceptual representations, we included every statement made by each participant in the present analyses. Each statement was coded by the first author with one of the 45 coding categories from a more recent version of $\mathrm{Wu}$ and Barsalou's (2009) coding scheme. This scheme has been recognized as the best method available for classifying features (McRae \& Cree, 2002). To establish reliability, the second author coded the statements for 1 participant's 11 concepts; interrater agreement reached $90 \%$.

The 45 coding categories fell into five general groups: taxonomic, property, setting, introspective, and miscellaneous. ${ }^{3}$ A taxonomic code was applied to statements that mentioned a taxonomic category related to the target concept (e.g., car, <vehicle $>$; furniture, $<$ sofa $>$ ). A property code was applied to statements that described a property of a physical object (e.g., apple $<$ seeds $>$; bird, $<$ hollow bones $>$ ). Statements that described features of situations were coded by setting and introspective codes. A setting code was applied to statements that described a feature of a setting or event (e.g., hammer, <found in a toolbox $>$; vegetable, <can be bought at the supermarket $>$ ), and an introspective code was applied to statements that described the mental state of an agent in a situation (e.g., salmon, $<$ tastes good $>$; insect, $<$ frightening $>$ ). Finally, a miscellaneous code was applied to statements reflecting aspects of the knowledge retrieval process (e.g., $<$ I do not know much about this $>$; <nothing else comes to mind right away $>$ ). For definitions of all general and specific coding categories, see Wu and Barsalou (2009).

Following Barsalou and Wiemer-Hastings (2005), we excluded the statements in the miscellaneous coding category from the analyses. In addition, all the concepts were included in the analyses to follow, except those used for training. Given that there was no effect of concept presentation order on the features retrieved $[F(28,28)=1.06$, $\left.p=.44, \eta_{\mathrm{p}}^{2}=.51\right]$, this variable was not included in the analyses reported below.
Table 1

Raw Frequencies of Feature Types Retrieved in the Open and Property Probe Conditions of Experiment 1

\begin{tabular}{lccccc}
\hline \multirow{2}{*}{ Probe } & \multicolumn{4}{c}{ Feature Types } & \\
\cline { 2 - 5 } & Taxonomic & Property & Setting & Introspective & Total \\
\hline Open & 2.86 & 6.64 & 7.57 & 2.71 & 4.33 \\
Property & 2.25 & 11.94 & 7.56 & 1.38 & 5.14 \\
\hline
\end{tabular}

Is the open probe able to elicit situational knowledge better than the property probe? Table 1 displays the raw frequencies of feature types retrieved in the open and property probe conditions. The numbers correspond to the mean of the participants' medians of features retrieved in each coding category. First, these numbers show that the property probe had a slight advantage over the open probe in terms of the total number of features elicited. Second, both probes elicited more properties and setting features, as compared with taxonomic and introspective features. Third, the largest difference between the retrieval patterns of the two probe conditions concerned the amount of properties retrieved.

In line with Barsalou and Wiemer-Hastings (2005), the main analyses were performed on the proportions computed over the four coding categories. Figures $1 \mathrm{~A}$ and $1 \mathrm{~B}$ plot the mean proportions of feature types retrieved in the open and property probe conditions, respectively. Figures $1 \mathrm{C}$ and $1 \mathrm{D}$ plot the corresponding data for Experiment 2 . The analyses reported in this section are based on Figures 1A and 1B; Figures 1C and 1D will be analyzed later, in the section pertaining to Experiment 2. Since our interest was in the patterns of mean proportions of feature types, we used within-subjects confidence intervals (CIs) in these figures. We adopted Masson and Loftus's (2003) procedure for constructing within-subjects CIs.

Figures $1 \mathrm{~A}$ and $1 \mathrm{~B}$ suggest that object concepts differentially emphasize different kinds of situational information. The proportion of setting features retrieved was larger than the proportion of introspective features in both probe conditions $[t(6)=-5.79, p<.01, r=.92 ; t(7)=-8.95$, $p<.01, r=.96$, for the open and property probe conditions, respectively]. ${ }^{4}$ This observation is consistent with the findings reported by Barsalou and Wiemer-Hastings (2005) for a smaller set of object concepts.

Did the retrieval pattern of properties and setting features differ between the probe conditions? Barsalou and WiemerHastings's (2005) reasoning suggests that the open probe should yield a larger proportion of setting features than of properties. The results displayed in Figure 1A do not support this hypothesis $[t(6)=-1.18, p=.28, r=.43]$. On the other hand, it follows from these authors' reasoning that the property probe should yield a larger proportion of properties than of setting features. The results displayed in Figure $1 \mathrm{~B}$ clearly support this hypothesis $[t(7)=4.47, p<$ $.01, r=.86]$. Taken as a whole, these results suggest that whereas the property probe boosted retrieval of properties, relative to setting features, the open probe did not have the corresponding effect on setting features. Notwithstanding this result, it could be that the open probe yielded a higher proportion of setting features, as compared with the prop- 
A

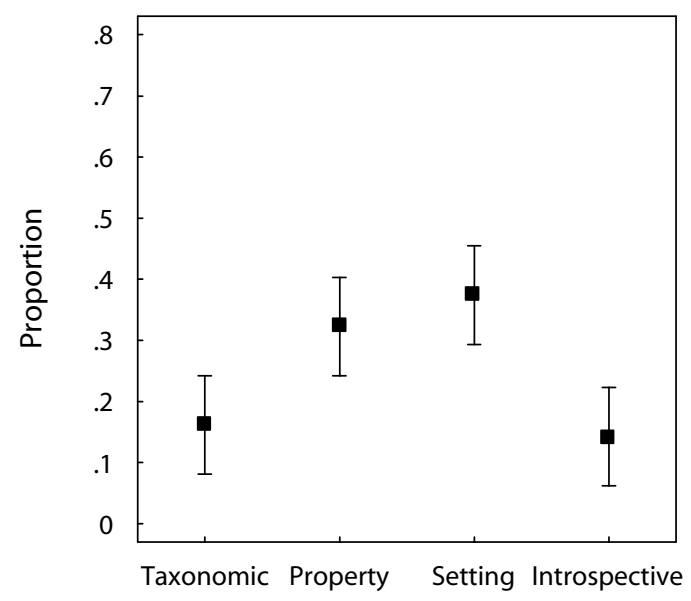

C Open Probe

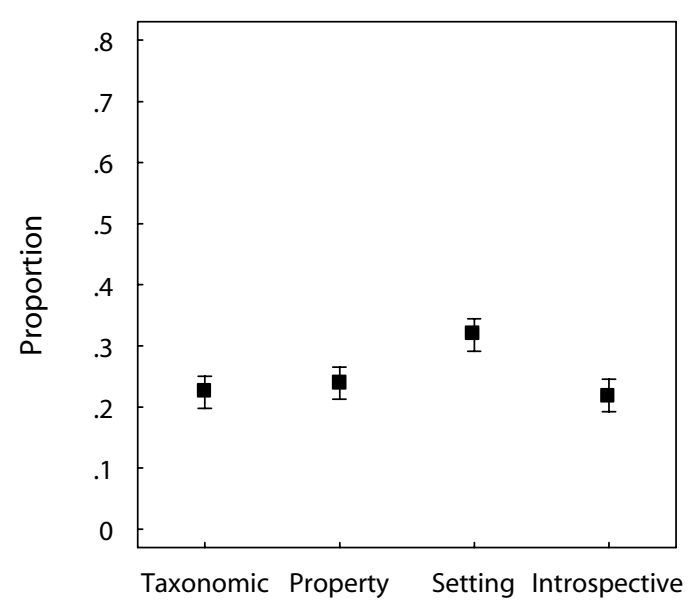

B Property Probe

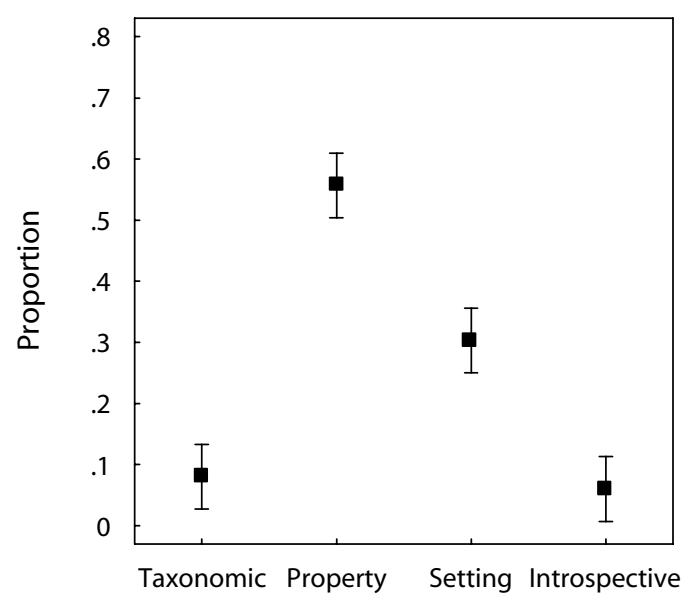

D Property Probe

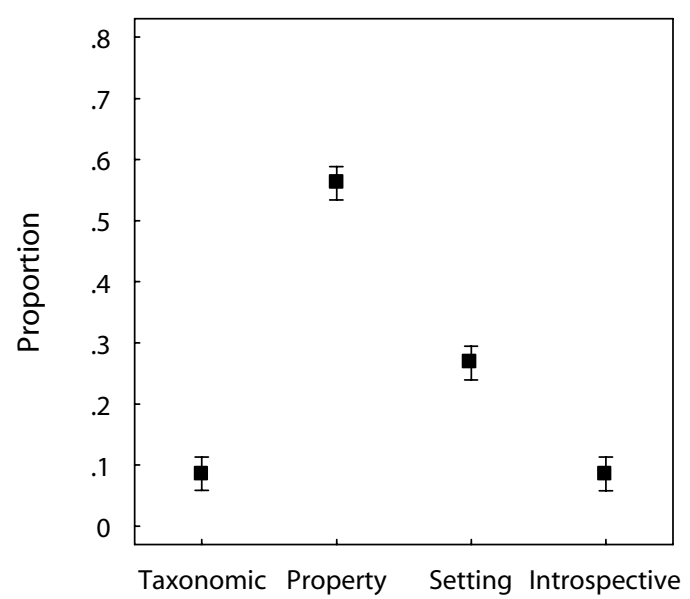

Figure 1. Mean proportions of feature types retrieved in Experiments 1 and 2 with $95 \%$ confidence intervals. Panels $A$ and $B$ plot the data from Experiment 1, for the open and property probe conditions, respectively. Panels $C$ and $D$ plot the data from Experiment 2, for the open and property probe conditions, respectively.

erty probe. The results suggest that this was not the case $[t(13)=2.08, p=.06, r=.50]$. In addition, the open probe did not yield an advantage with respect to features in the taxonomic $[t(13)=2.02, p=.07, r=.49]$ and introspective $[t(13)=2.10, p=.06, r=.50]$ categories, with the property probe eliciting a significantly higher proportion of properties $[t(13)=-4.94, p<.01, r=.81]$.

All in all, but with the caveat of a potential lack of statistical power, these results suggest that the open probe was not, in general, better able to elicit situational knowledge, as compared with the property probe. First, the open probe did not boost retrieval of setting features, relative to properties - a result that is inconsistent with that reported by Barsalou and Wiemer-Hastings (2005) for a smaller set of object concepts. Second, the open probe elicited the same proportion of setting and introspective features as the property probe.
How do superordinate and basic concepts differ in content? In line with Murphy and Wisniewski's (1989) logic, we expected the proportion of setting features retrieved for superordinates to be larger than the proportion of properties, with the opposite pattern being observed for basic concepts. In the open probe condition, the same proportions of properties and setting features were retrieved for both superordinate $[t(2)=-1.29, p=$ $.33, r=.99]$ and basic $[t(3)=-0.40, p=.72, r=.22]$ concepts. In the property probe condition, on the other hand, an advantage of properties over setting features was observed for basic concepts $[t(3)=4.18, p=.03, r=1]$, but, for superordinates, the corresponding advantage did not reach the conventional level of significance $[t(3)=$ $2.71, p=.07, r=1]$. All in all, the relative proportions for properties and setting features were more or less identical for the two concept levels in each probe condition, a 
result that does not support the hypothesis derived from Murphy and Wisniewski.

\section{EXPERIMENT 2}

The low number of participants who took part in Experiment 1 raises the possibility that the analyses reported above lack statistical power. In fact, only 15 participants were included in a $2 \times 2$ between-subjects design. Hence, a second experiment was conducted with the purpose of replicating Experiment 1, using a larger sample of participants.

\section{Method}

Participants and Design. One hundred twenty-three participants, with an average age of 25 years, were assigned to one of four between-subjects conditions varying the retrieval probe and the taxonomic level of the concept. For 23 participants, an error in the program caused part of the data to be lost. Hence, these participants were excluded from the analyses reported in the next section. All the participants were native German speakers and were paid $€ 10$ for taking part in the study.

Materials. The two sets of 11 superordinate and 11 basic concepts used in Experiment 1 were used as stimuli (i.e., reptile, amphibian, mammal, fish, insect, tool, vegetable, fruit, bird, vehicle, and furniture as the superordinate set, and snake, frog, dog, salmon, fly, hammer, lettuce, apple, sparrow, car, and sofa as the basic set). In addition, three extra concepts were included in each set (appliance, clothing, and weapon in the superordinate set; refrigerator, pants, and pistol in the basic set). Including these items allowed for better counterbalancing the number of natural kinds and artifacts in the two concept sets. Finally, two training concepts were used, the same as those employed in the training phase of Experiment 1 (musical instrument and flower in the superordinate condition; guitar and tulip in the basic condition). Overall, the concepts employed in this experiment were those that had been used in studies looking into the nature of superordinate- and basic-level semantic knowledge (e.g., Marques, 2007; Rosch \& Mervis, 1975; Rosch, Mervis, Gray, Johnson, \& Boyes-Braem, 1976).

Procedure. A computerized version of the experiment was developed that corresponded closely to the verbal knowledge production in Experiment 1. The main difference was that the responses were typed rather than said aloud. The participants were first presented with the task instructions on the computer screen. Half of the participants received the property probe, and the other half received the open probe. The probes were again formulated as described in the Appendix.

In this experiment, the participants in the open probe condition were asked to describe their thoughts in complete sentences. Whereas in Experiment 1 the participants in this condition naturally produced complete sentences, pilot sessions in Experiment 2 revealed that participants tended to type in isolated words instead (e.g., reptile, < $<$ green $>$ ). Responses composed of isolated words cannot be unambiguously coded, since their relation to the target concept is left unspecified. In the example given, the participant may have either thought that reptiles are green (with $<$ green $>$ being a property) or that they live in green areas (with $<$ green $>$ being a setting feature). To make data coding as unambiguous as possible and to better mimic the task of producing knowledge verbally, the participants were asked to describe their thoughts in complete sentences so that a person who would later read their responses would know exactly what they were thinking about.

Once the instructions had been given, the participants retrieved knowledge either for a series of superordinate concepts or for a series of basic concepts. The participants in both probe conditions first practiced retrieving knowledge for two superordinate or two basic concepts. After training, the participants retrieved knowledge for 14 test items, presented in random order.

To better capture the flavor of the verbal production that characterized Experiment 1, the participants in both probe conditions were not shown their previous responses. The program stored each statement and deleted it from the screen before the next response was typed in.

The time to work on each concept was restricted to $4 \mathrm{~min}$. A time bar was presented on the screen, so that the participants knew how much time was left for them to work on each concept. However, if the participants did not type a response for $30 \mathrm{sec}$, the program would move automatically to the next concept even before $4 \mathrm{~min}$ had elapsed. This prevented the participants from having to wait for the $4 \mathrm{~min}$ to elapse, when no further thoughts or properties were coming to mind. Finally, there was a pause of $5 \mathrm{sec}$ between concepts. The participants taking part in pilot sessions of the experiment reported that these time frames allowed them to comfortably perform the task.

On every trial, depending on the condition, the participants were asked to list the properties of the target concept or to report their thoughts about it. The instructions given on each trial were equivalent to those given verbally by the experimenter on every trial in Experiment 1.

\section{Results and Discussion}

The participants' responses were coded directly from German with one of the five general coding categories from Wu and Barsalou's (2009) coding scheme by a native German speaker who was naive as to the purpose of the study. To establish reliability, the first author coded the data of 16 participants, 4 belonging to each of the four between-subjects conditions. Interrater agreement reached $93 \%$. The concepts used for training and the statements in the miscellaneous coding category were excluded from the analyses reported below.

Is the open probe able to elicit situational knowledge better than the property probe? The raw frequencies of feature types presented in Table 2, corresponding to the means of the participants' medians for features retrieved in each coding category, display more or less the same pattern as the raw data shown in Table 1. Nevertheless, the values displayed in Table 2 are lower overall, perhaps because the time to work on each concept was restricted to 4 min in Experiment 2, whereas it was unlimited in Experiment 1. In addition, responses were typed in Experiment 2 but were given verbally in Experiment 1 .

As for Experiment 1, we performed the main analyses on the proportions computed over the four coding categories. Figures $1 \mathrm{C}$ and $1 \mathrm{D}$ show the mean proportions of feature types retrieved in the two probe conditions in Experiment 2. These results replicate the finding that object concepts put a greater emphasis on setting features, as compared with introspective features. The proportion

Table 2

Raw Frequencies of Feature Types Retrieved in the Open and Property Probe Conditions of Experiment 2 Feature Types

\begin{tabular}{lccccc}
\cline { 2 - 5 } Probe & Taxonomic & Property & Setting & Introspective & Total \\
\hline Open & 2.11 & 2.25 & 3.44 & 2.30 & 2.57 \\
Property & 0.89 & 6.78 & 3.16 & 0.80 & 2.93 \\
\hline
\end{tabular}


of setting features retrieved was larger than the proportion of introspective features in both probe conditions $[t(49)=4.85, p<.01, r=.57$, for the open probe condition; $t(49)=10.32, p<.01, r=.83$, for the property probe condition].

Figures $1 \mathrm{C}$ and $1 \mathrm{D}$ further suggest that the retrieval pattern of properties and setting features differed between the probe conditions. In line with Experiment 1, the property probe yielded a larger proportion of properties than of setting features $[t(49)=8.19, p<.01, r=.76]$. However, the difference between the proportion of setting features and properties retrieved in the open probe condition reached significance in this experiment $[t(49)=-3.77$, $p<.01, r=.47]$. The latter result replicates that reported by Barsalou and Wiemer-Hastings (2005) for a smaller set of object concepts. Furthermore, the open probe yielded higher proportions of setting $[t(98)=2.28, p=.03, r=$ $.22]$, introspective $[t(98)=9.68, p<.01, r=.70]$, and taxonomic $[t(98)=7.59, p<.01, r=.61]$ features, as

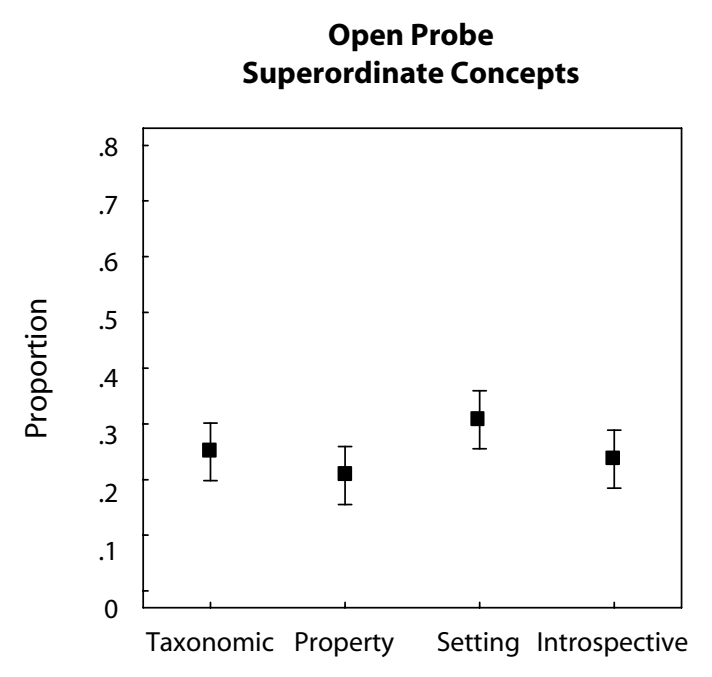

\section{Open Probe \\ Basic Concepts}

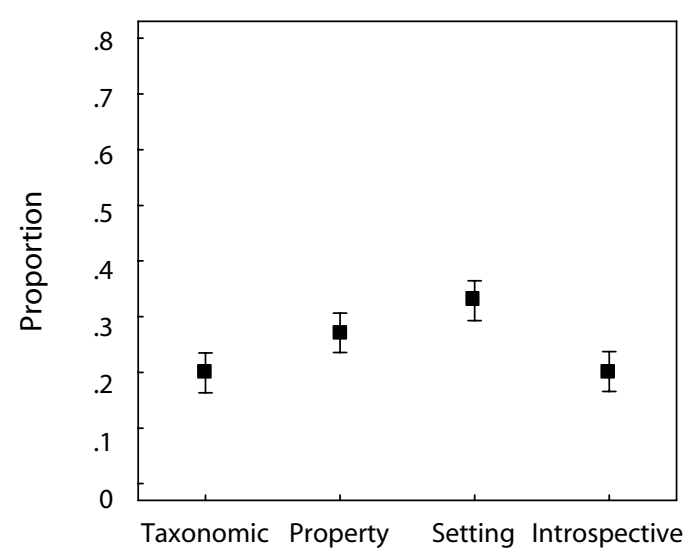

compared with the property probe. None of these differences reached the conventional level of significance in Experiment 1. On the other hand, the result concerning the proportion of properties retrieved matched that observed in Experiment 1. Once again, the open probe elicited a lower proportion of properties, as compared with the property probe $[t(98)=-13.15, p<.01, r=.80]$.

As Figure 1 clearly shows, the pattern of results observed in Experiment 2 is, in general, highly consistent with that observed in Experiment 1. The only difference was that, in Experiment 2, the open probe was better able to elicit situational knowledge than was the property probe, in support of Barsalou and Wiemer-Hastings's (2005) line of reasoning. Not only did the open probe boost retrieval of setting features, as compared with properties, but it also produced higher proportions of setting and introspective features, as compared with the property probe. It appears that the open probe did not have the same advantage in Experiment 1, due to a lack of statistical power.
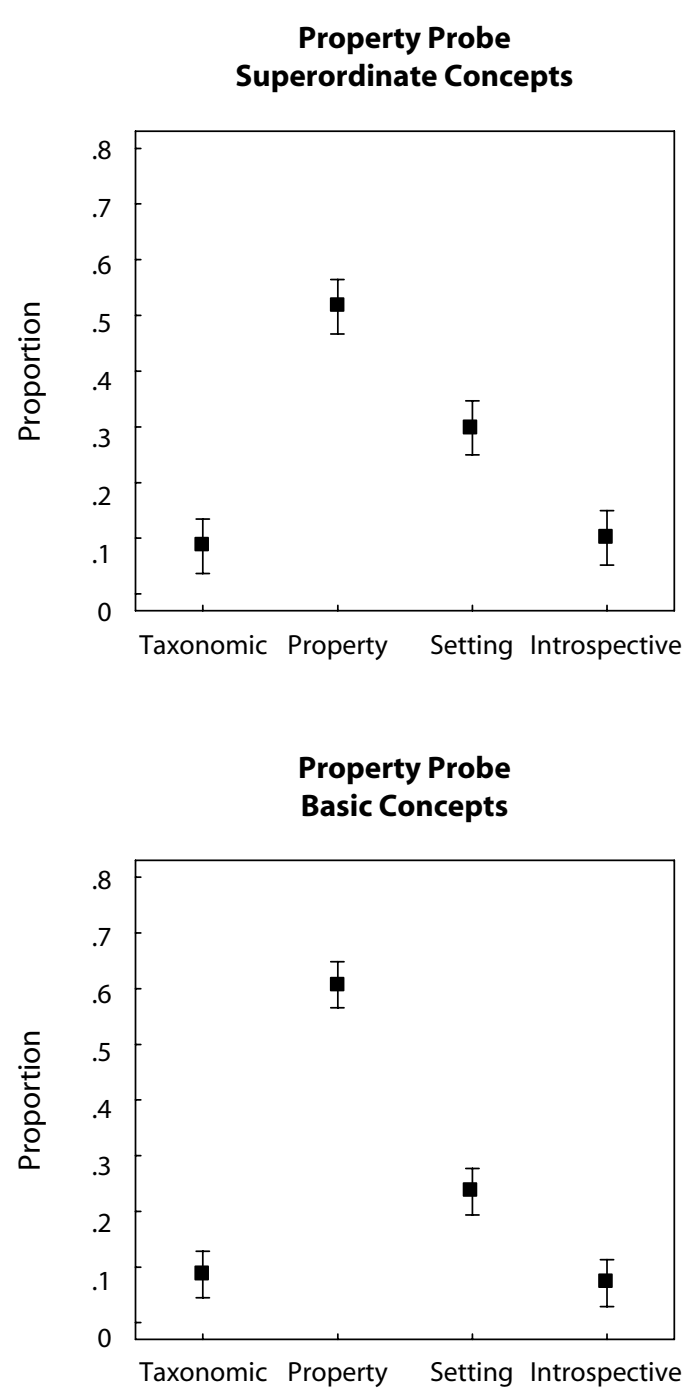

Figure 2. Mean proportions of feature types retrieved for superordinate and basic concepts in the open and property probe conditions in Experiment 2 with $95 \%$ confidence intervals. 
How do superordinate and basic concepts differ in content? Figure 2 presents the mean proportions of feature types retrieved for superordinate and basic concepts (upper and lower panels, respectively) in the open and property probe conditions (left- and right-side panels, respectively) in Experiment 2. A first result is that the participants retrieved more properties for basic concepts than for their superordinates in both probe conditions $[t(48)=$ $-2.40, p=.02, r=.36 ; t(48)=-2.38, p=.02, r=.35$, for the open and property probe conditions, respectively]. This advantage of basic concepts over superordinates in terms of the properties retrieved is a well-documented finding in the concepts literature (e.g., Rosch et al., 1976; Tversky \& Hemenway, 1984). Second, Figure 2 shows that the proportion of setting features did not differ between concept levels in both probe conditions, nor did the proportions of taxonomic and introspective features.

In line with Murphy and Wisniewski's (1989) logic, we expected the proportion of setting features retrieved for superordinates to be larger than the proportion of properties, with the opposite pattern being observed for basic concepts. Figure 2 shows that, in the open probe condition, a higher proportion of setting features was retrieved over properties for both concept levels $[t(24)=-3.06, p<.01, r=.53$; $t(24)=-2.21, p=.04, r=.41$, for superordinate and basic concepts, respectively]. This difference did not reach the conventional level of significance in Experiment 1. In the property probe condition, as in Experiment 1, an advantage of properties over setting features was observed for both concept levels $[t(24)=4.18, p<.01, r=.65$; $t(24)=8.14, p<.01, r=.86$, for superordinate and basic concepts, respectively]. Together with the findings of Experiment 1 , these results provide evidence counter to the hypothesis, inspired by Murphy and Wisniewski (1989), concerning the differential focus of superordinate and basic concepts on properties and setting features.

Nevertheless, the data displayed in Figure 2 appear to be more consistent with an alternative interpretation of Murphy and Wisniewski's (1989) proposal. Our initial interpretation was that superordinates may put more emphasis on setting information than on properties, with the reverse being true for basic concepts. Alternatively, superordinates may simply put more weight on setting features, relative to properties, than basic concepts do. The differential weighing of setting features, as compared with properties, can be verified in terms of the magnitude of the difference between the corresponding proportions for superordinate and basic concepts, in each probe condition.

In the open probe condition, as was observed earlier, the proportion of setting features retrieved was higher than the proportion of properties for either concept level. Yet the difference between the two proportions was somewhat larger for superordinates than for basic concepts, albeit below the conventional level of significance $[t(48)=$ $-1.01, p=.32, r=.99]$. In the property probe condition, on the other hand, we observed that the proportion of properties retrieved was higher than the proportion of setting features for both concept levels. Yet the difference between the two proportions was larger for basic concepts than for their superordinates $[t(48)=-2.23, p=.03$, $r=.99]$. These results are in line with the alternative interpretation that superordinates put more weight on setting features than basic concepts do. In the open probe condition, superordinates display a slightly larger retrieval advantage of setting features over properties, as compared with basic concepts. In the property probe condition, on the other hand, superordinates display a smaller advantage of properties over setting features.

\section{CONCLUSION}

This work substantiates the view that concepts are situated representations, whose diverse content is retrieved even when the probe restricts responses to their defining properties. Our primary goal was to evaluate Barsalou and Wiemer-Hastings's (2005) proposal that open-ended response collection is a better method for eliciting the situational content of concepts, as compared with a probe that restricts responses. The findings of Experiment 1 did not back up this proposal, yet the performed analyses may have lacked statistical power due to a small number of participants. Experiment 2, on the contrary, provided convincing support for Barsalou and Wiemer-Hastings's claim. Not only did the open probe boost retrieval of setting features, relative to properties, but it also elicited higher proportions of setting and introspective features, as compared with the property probe. Except for these two pieces of evidence, the findings of Experiment 2 followed those of the first experiment. As Figure 1 shows, the overall patterns of the two experiments are remarkably similar despite the differences in the methods used. Whereas in Experiment 1, the participants produced knowledge verbally for as long as they could, in Experiment 2, they were asked to type their responses within a fixed time limit.

Finally, despite the disadvantage of the property probe in eliciting situational content, we believe that it might still be a useful tool for collecting semantic feature production norms. From a practical point of view, responses to the property probe are easier to code, due to their constrained format. Our recommendation for studies norming concepts is to use the property probe in combination with a setting probe (e.g., "describe the settings in which snakes occur") and an introspective probe (e.g., "report what you feel about snakes"). This methodological procedure would have the value of increasing the proportion of setting and introspective features elicited by the property probe, while allowing for a simple response-coding procedure. Finally, the property probe appears to be as suitable as the open probe for exploring the content of concepts differing in taxonomic generality. Both probes produced a basic-level advantage in terms of the proportion of properties retrieved, with no differences being observed for the remaining feature types.

\section{AUTHOR NOTE}

The authors thank Soo-Youn Lee for coding the data in Experiment 2 and Lawrence Barsalou for providing the updated version of $\mathrm{Wu}$ and Barsalou's (2009) coding scheme. This work was supported by the Max Planck Society and a fellowship to A.S.M. from the International Max Planck Research School "The Life Course: Evolutionary and Ontogenetic Dynamics (LIFE)." The authors thank the fellows and faculty of LIFE and the members of the Center for Adaptive Behavior and Cognition for help- 
ful comments and discussions. Correspondence concerning this article should be addressed to A. S. Morais, Center for Adaptive Behavior and Cognition, Max Planck Institute for Human Development, Lentzeallee 94, 14195 Berlin, Germany (e-mail: morais@mpib-berlin.mpg.de).

\section{Note-Accepted by the previous editorial team, when John H. Krantz was Editor.}

\section{REFERENCES}

Barsalou, L. W. (2003). Abstraction in perceptual symbol systems. Philosophical Transactions of the Royal Society B, 358, 1177-1187.

Barsalou, L. W., \& Wiemer-Hastings, K. (2005). Situating abstract concepts. In D. Pecher \& R. A. Zwaan (Eds.), Grounding cognition: The role of perception and action in memory, language, and thinking (pp. 129-163). New York: Cambridge University Press.

CaLlanan, M. A. (1985). How parents label objects for young children: The role of input in the acquisition of category hierarchies. Child Development, 56, 508-523. doi:10.2307/1129738

Garrard, P., Lambon Ralph, M. A., Hodges, J. R., \& Patterson, K. (2001). Prototypicality, distinctiveness, and intercorrelation: Analyses of the semantic attributes of living and nonliving concepts. Cognitive Neuropsychology, 18, 125-174. doi:10.1080/02643290042000053

Marques, J. F. (2007). The general/specific breakdown of semantic memory and the nature of superordinate knowledge: Insights from superordinate and basic-level feature norms. Cognitive Neuropsychology, 24, 879-903. doi:10.1080/02643290701789436

Masson, M. E. J., \& Loftus, G. R. (2003). Using confidence intervals for graphically based data interpretation. Canadian Journal of Experimental Psychology, 57, 203-220. doi:10.1037/h0087426

McRAE, K., \& CREE, G. S. (2002). Factors underlying category-specific semantic deficits. In E. M. E. Forde \& G. W. Humphreys (Eds.), Category specificity in brain and mind (pp. 211-249). Hove, U.K.: Psychology Press.

McRae, K., Cree, G. S., Seidenberg, M. S., \& McNorgan, C. (2005). Semantic feature production norms for a large set of living and nonliving things. Behavior Research Methods, Instruments, \& Computers, 37, 547-559.

MurPhy, G. L., \& WisniewsKi, E. J. (1989). Categorizing objects in isolation and in scenes: What a superordinate is good for. Journal of Experimental Psychology: Learning, Memory, \& Cognition, 15, 572586. doi:10.1037/0278-7393.15.4.572

Rosch, E., \& Mervis, C. B. (1975). Family resemblances: Studies in the internal structure of categories. Cognitive Psychology, 7, 573-605. doi:10.1016/0010-0285(75)90024-9

Rosch, E., Mervis, C. B., Gray, W. D., Johnson, D. M., \& BoyesBraem, P. (1976). Basic objects in natural categories. Cognitive Psychology, 8, 382-439. doi:10.1016/0010-0285(76)90013-X

Ruts, W., De Deyne, S., Ameel, E., Vanpaemel, W., Verbeemen, T., \& STORMS, G. (2004). Dutch norm data for 13 semantic categories and 338 exemplars. Behavior Research Methods, Instruments, \& Computers, 36, 506-515.

TVersky, B., \& Hemenway, K. (1984). Objects, parts, and categories. Journal of Experimental Psychology: General, 113, 169-193. doi:10.1037/0096-3445.113.2.169

WisNiewsKi, E. J., \& MuRPHY, G. L. (1989). Superordinate and basic category names in discourse: A textual analysis. Discourse Processes, 12, 245-261.

Wu, L.-L., \& Barsalou, L. W. (2009). Perceptual simulation in conceptual combination: Evidence from property generation. Acta Psychologica, 132, 173-189.

Yeh, W., \& Barsalou, L. W. (2006). The situated nature of concepts. American Journal of Psychology, 119, 349-384.

\section{NOTES}

1. The term properties is used to denote the intrinsic characteristics exemplars may possess, and the term features is employed to refer to all types of information a concept may be associated with.

2. There are also concepts more specific than basic concepts, such as convertible or python, which are called subordinates, but we do not consider these here.

3. The coding category property is called entity in Barsalou and Wiemer-Hastings (2005) and Wu and Barsalou (2009). The coding category setting is called setting/event in Barsalou and Wiemer-Hastings and situation in $\mathrm{Wu}$ and Barsalou.

4. We report exact $p$ values throughout these analyses, but when they fall below .01, we report them as $<.01$.

\section{APPENDIX}

Open Probe

(Adapted From Barsalou \& Wiemer-Hastings, 2005)

\section{German Version}

Bei diesem Experiment möchten wir herausfinden, wie Menschen spezifische Begriffe verstehen. Bitte beachten Sie, dass es bei der folgenden Aufgabe keine richtigen oder falschen Antworten gibt. Machen Sie sich daher bitte keine Gedanken, ob Sie eine richtige Antwort gegeben haben oder nicht. Das ist nicht relevant für uns. Uns interessiert, wie Menschen wie Sie über verschiedene Begriffe denken. Sie können uns folgendermaßen dabei helfen, mehr über dieses Thema zu erfahren. In dem Moment, in dem ich Ihnen bestimmte Begriffe nenne, antworten Sie bitte mit den ersten Gedanken, die Ihnen in den Sinn kommen. [In Experiment 2, the latter sentence was adapted to the written response format: Wir werden Ihnen gleich eine Folge von einfachen Begriffen präsentieren. Bitte lesen Sie jeden Begriff aufmerksam und schreiben hierzu die ersten Gedanken auf, die Ihnen in den Sinn kommen.] Anschließend fahren Sie mit den weiteren Gedanken, die Ihnen einfallen fort.

\section{English Translation}

Our specific purpose here today is to learn more about how people understand a few specific concepts. Let me stress that there are no correct or incorrect responses to the questions that I am about to ask you. Thus, please do not worry about whether you have come up with the right answer or not. This is not an issue at all. Instead, we are interested in how people like you think about various concepts. Here is what you can do to help us learn more about this. In a moment, when I ask you about various concepts, please respond with the very first thoughts that come to mind. [In Experiment 2, the latter sentence was adapted to the written response format: In a moment, you will be presented with a series of simple concepts. Please read each concept and type in the very first thoughts that come to mind.] Then keep responding with the thoughts that continue to come to mind. 


\section{APPENDIX (Continued)}

Property Probe

German Version

(Adapted From Garrard et al., 2001)

Bei diesem Experiment möchten wir herausfinden, wie Menschen spezifische Begriffe verstehen. Sie können uns folgendermaßen dabei helfen, mehr über dieses Thema zu erfahren. Gleich werde ich Sie bitten, die definierende Eigenschaften eines Begriffs zu nennen. [In Experiment 2, the latter sentence was adapted to the written response format: Wir werden Ihnen gleich eine Folge von einfachen Begriffen präsentieren. Bitte lesen Sie jeden Begriff aufmerksam und schreiben Sie dessen Eigenschaften auf, die ihn definieren.] Die Eigenschaften können aus einzelnen Wörtern oder aus kurzen Sätzen bestehen, die mit dem Begriff über die Worte "ist", "hat" oder "kann" in Bezug stehen. "Ist" Eigenschaften sind beobachtbare oder nicht beobachtbare Charakteristika, die den Gegenstand beschreiben. "Hat" Eigenschaften beziehen sich auf Teile des Gegenstandes und "kann" Eigenschaften auf die Fähigkeiten, Tätigkeiten und den Gebrauch des Gegenstandes.

\section{English Translation}

Our specific purpose here today is to learn more about how people understand a few specific concepts. Here is what you can do to help us learn more about this. In a moment, I will ask you to report the defining properties of a few concepts. [In Experiment 2, the latter sentence was adapted to the written response format: In a moment, you will be presented with a series of simple concepts. Please read each concept and type in its defining properties.] The properties should consist of single words or short phrases that could be connected to the concept by the words "is," "has," or "can." "Is" properties are observable and nonobservable characteristics that describe the item, "has" properties refer to parts of the item, and "can" properties to the abilities, activities, and uses of the item.

(Manuscript received December 7, 2008;

revision accepted for publication September 25, 2009.) 